\title{
Overexpression of endothelin B receptor in glioblastoma: a prognostic marker and therapeutic target?
}

\author{
Suhas Vasaikar ${ }^{1}$, Giorgos Tsipras ${ }^{1}$, Natalia Landázuri ${ }^{1}$, Helena Costa ${ }^{2}$, Vanessa Wilhelmi ${ }^{2}$, Patrick Scicluna ${ }^{2}$, \\ Huanhuan L. Cui ${ }^{2}$, Abdul-Aleem Mohammad ${ }^{2}$, Belghis Davoudi ${ }^{2}$, Mingmei Shang ${ }^{1}$, Sharan Ananthaseshan², \\ Klas Strååt ${ }^{3}$, Giuseppe Stragliotto ${ }^{4}$, Afsar Rahbar ${ }^{2}$, Kum Thong Wong ${ }^{5}$, Jesper Tegner ${ }^{1,6}$, Koon-Chu Yaiw ${ }^{2^{*}}$ (D) \\ and Cecilia Söderberg-Naucler ${ }^{2^{*}}$
}

\begin{abstract}
Background: Glioblastoma (GBM) is the most common malignant brain tumor with median survival of 12-15 months. Owing to uncertainty in clinical outcome, additional prognostic marker(s) apart from existing markers are needed. Since overexpression of endothelin B receptor (ETBR) has been demonstrated in gliomas, we aimed to test whether ETBR is a useful prognostic marker in GBM and examine if the clinically available endothelin receptor antagonists (ERA) could be useful in the disease treatment.

Methods: Data from The Cancer Genome Atlas and the Gene Expression Omnibus database were analyzed to assess ETBR expression. For survival analysis, glioblastoma samples from 25 Swedish patients were immunostained for ETBR, and the findings were correlated with clinical history. The druggability of ETBR was assessed by protein-protein interaction network analysis. ERAs were analyzed for toxicity in in vitro assays with GBM and breast cancer cells.

Results: By bioinformatics analysis, ETBR was found to be upregulated in glioblastoma patients, and its expression levels were correlated with reduced survival. ETBR interacts with key proteins involved in cancer pathogenesis, suggesting it as a druggable target. In vitro viability assays showed that ERAs may hold promise to treat glioblastoma and breast cancer.
\end{abstract}

Conclusions: ETBR is overexpressed in glioblastoma and other cancers and may be a prognostic marker in glioblastoma. ERAs may be useful for treating cancer patients.

Keywords: Glioblastoma, Endothelin B receptor, Endothelin receptor antagonists

\section{Background}

Glioblastoma (GBM; World Health Organization grade IV astrocytoma) is the most common malignant brain tumor with an annual incidence of 3.5 cases per 100,000 worldwide [1]. It is also one of the most lethal human cancers. The median overall survival is $12-15$ months with standard treatment [2], and 3-6 months for

\footnotetext{
*Correspondence: ykcywx@yahoo.com; Cecilia.Naucler@ki.se

Suhas Vasaikar, Giorgos Tsipras and, Natalia Landázuri are contributed equally.

Koon-Chu Yaiw and Cecilia Söderberg-Naucler are senior author.

${ }^{2}$ Cell and Molecular Immunology, Experimental Cardiovascular Unit,

Departments of Medicine and Neurology, Center for Molecular Medicine,

Karolinska Institutet, SE-171 76 Stockholm, Sweden

Full list of author information is available at the end of the article
}

patients with recurrent GBM [3]. Owing to uncertainty in clinical outcome in individual patients, new prognostic markers are needed for GBM patients, especially those with potential to affect patient outcome through druggable targets.

Endothelins are vasoactive peptides that exert their effects through interactions with the G-protein-coupled receptors endothelin receptor A (ETAR) and endothelin receptor B (ETBR). ETAR is expressed mainly in vascular smooth muscle cells and stromal cells, whereas ETBR is expressed mainly in endothelial cells; ETAR mediates vasoconstriction, and ETBR vasodilatation and also stimulates cell proliferation (reviewed in [4]. Dysregulation of ETBR has been implicated in 
cardiovascular disease and linked to a congenital disorder, Hirschsprung's disease (reviewed in [4]). Moreover, ETBR is overexpressed in vulvar cancer [5], clear-cell renal cell carcinoma [6], and esophageal squamous cell carcinoma [7] and is closely associated with disease progression and poor patient survival [5-7]. Consistent with a crucial role for ETBR in tumorigenesis, some ETBR antagonists may be beneficial in treating melanoma or glioma [8-11].

Overexpression of ETBR in GBM was associated with a poor prognosis in a Chinese population [12]. Since ethnicity may play a major role in the pathogenesis of gliomas [13, 14], we investigated whether ETBR overexpression could be detected in patients with GBM and in other cancers outside of China, whether ETBR expression correlates with patient survival (and thus its potential use as a prognostic marker and/or therapeutic target), and whether clinically available endothelin receptor blockers/antagonists have toxic effects on cancer cells in vitro.

\section{Methods}

Patient cohort

Formalin-fixed, paraffin-embedded tissue sections were from $25 \mathrm{GBM}$ cases, were selected from our previously studied cohort without prior selection [15]. Demographic information and clinical data with time to tumor progression (TTP) and overall survival (OS) for all GBM cases are shown in Table 1. Ten normal samples from aging control brains (frontal part of brain from men) median age 57 [50-61 yrs] were from the Department of Pathology, University of Malaya Medical Center (ethical number 896.7). The use of patient materials was approved by the Ethics Committee at the Karolinska Institutet and by the Medical Ethics Committee, University of Malaya Medical Center, Malaysia, and conducted in accordance with the Declaration of Helsinki.

\section{ETBR immunohistochemistry}

Formalin-fixed, paraffin-embedded sections were analyzed by immunohistochemistry as described but with minor

Table 1 Demographic and available clinical information

\begin{tabular}{|c|c|c|c|c|c|c|c|}
\hline \multirow{2}{*}{$\begin{array}{l}\text { Case } \\
\text { number }\end{array}$} & \multirow{2}{*}{$\begin{array}{l}\text { TTP } \\
\text { (months) }\end{array}$} & \multirow{2}{*}{$\begin{array}{l}\text { OS } \\
\text { (months) }\end{array}$} & \multirow{2}{*}{$\begin{array}{l}\text { ETBR } \\
\text { expression }\end{array}$} & \multirow{2}{*}{$\begin{array}{l}\text { Age } \\
\text { (years) }\end{array}$} & \multirow[t]{2}{*}{ Gender } & \multicolumn{2}{|c|}{ Extent of resection } \\
\hline & & & & & & Radical & Partial \\
\hline K7686-2004 & 4 & 5 & $3+$ & 73 & M & No & Yes \\
\hline K9802-2004 & 5 & 5 & $1+$ & 68 & M & Yes & No \\
\hline K4448-2004 & 1 & 14 & $1+$ & 66 & M & No & Yes \\
\hline K12700-2004 & 1 & 5 & $1+$ & 64 & $\mathrm{~F}$ & Yes & No \\
\hline K10452-2004 & 7 & 10 & $1+$ & 59 & $\mathrm{~F}$ & Yes & No \\
\hline K5126-2004 & 3 & 7 & $1+$ & 57 & M & Yes & No \\
\hline K11136-2004 & 12 & 20 & $1+$ & 56 & M & Yes & No \\
\hline K17437-2004 & 16 & 17 & $2+$ & 56 & M & Yes & No \\
\hline K4840-2004 & 7 & 20 & $1+$ & 55 & M & Yes & No \\
\hline K16204-2004 & 10 & 11 & $1+$ & 54 & M & Yes & No \\
\hline K9236-2004 & 12 & 15 & $1+$ & 49 & $\mathrm{~F}$ & Yes & No \\
\hline K16178-2004 & 12 & 13 & $1+$ & 45 & M & Yes & No \\
\hline K3839-2004 & 12 & 14 & $3+$ & 28 & M & Yes & No \\
\hline K16102-2004 & 48 & 48 & $1+$ & 57 & $\mathrm{~F}$ & Yes & No \\
\hline K17407-2004 & 48 & 48 & $1+$ & 26 & $\mathrm{~F}$ & Yes & No \\
\hline K10315-2004 & 52 & 52 & $1+$ & 29 & M & Yes & No \\
\hline K3174-2004 & 15 & 19 & $3+$ & 79 & $\mathrm{~F}$ & Yes & No \\
\hline K16595/04 & 17 & 36 & $1+$ & 53 & $F$ & Yes & No \\
\hline K1716-2005 & 7 & 82 & $1+$ & 43 & M & Yes & No \\
\hline K3349-2005 & 3 & 3 & $3+$ & 79 & $\mathrm{~F}$ & No & Yes \\
\hline K8622-2005 & 9 & 12 & $3+$ & 59 & $\mathrm{~F}$ & Yes & No \\
\hline K9731-2005 & 4 & 7 & $3+$ & 52 & $F$ & Yes & No \\
\hline K15725-2005 & 2 & 4 & $2+$ & 54 & M & Yes & No \\
\hline K16886-2005 & 4.5 & 14.5 & $2+$ & 38 & $\mathrm{~F}$ & No & Yes \\
\hline K17972-2005 & 8.5 & 18 & $1+$ & 63 & $\mathrm{~F}$ & Yes & No \\
\hline
\end{tabular}

TTP time to tumor progression, OS overall survival; Staining was graded as low (1+) or high (2+ and $3+)$ 
modifications [7]. In brief, the sections were deparaffinized and rehydrated in a graded series of ethanol, and antigen was retrieved with the Decloaking Chamber NxGen (Biocare Medical, Concord, CA, USA) and Antigen Retrieval Citra Plus solution (Biogenex, Emergo Europe, The Hague, The Netherlands) at $110{ }^{\circ} \mathrm{C}$ for $15 \mathrm{~min}$. The sections were cooled to room temperature, equilibrated with Tris-buffered saline, $\mathrm{pH}$ 7.6, and subjected to a series of blocking steps with protein block (Dako Sweden, Stockholm, Sweden), Fc receptor blocker (Biogenex), and normal horse serum. The sections were then incubated with primary rabbit anti-ETBR (cat. no. E9905; 1:200, Sigma-Aldrich, Stockholm, Sweden) at $4{ }^{\circ} \mathrm{C}$ for $16 \mathrm{~h}$, washed three times with Tris-buffered saline, and placed in $3 \%(v / v) \mathrm{H}_{2} \mathrm{O}_{2}$ in water for $15 \mathrm{~min}$ at room temperature to quench endogenous peroxidase activity. After three washings with Tris-buffered saline, the sections were incubated with secondary anti-rabbit antibody conjugated to horseradish peroxidase (ImmPRESS kit, Vector Laboratories, Orton Southgate, Peterborough, UK). Immunoreactivity was revealed with diaminobenzidine (Innovex Biosciences, GENTAUR Europe BVBA, Belgium). The sections were then counterstained with hematoxylin, dried, and mounted with xylene-based mounting medium. Positive staining was graded as low or high as described [12].

\section{Analysis of data from the cancer genome atlas (TCGA) and the genome expression omnibus (GEO)}

To evaluate the ETBR expression profile in GBM patients, we obtained primary and processed gene expression data for TCGA GBM cohort from The Broad Institute TCGA GDAC Firehose (https://gdac.broadinstitute.org/) using RTCGA (http://rtcga.github.io/RTCGA). Kaplan-Meier survival curves were plotted using the clinical information submitted for GBM patients in TCGA. GEO datasets related to GBM (GSE2223, GSE7696, GSE16011, GSE10878, GSE46016, GSE15824, GSE31262, GSE42656, and GSE50161) were analyzed for ETBR expression normalized to control. Among selected studies GSE2223 (origin: normal brain samples and glioblastoma), GSE7696 (origin: non-tumoral brain samples and glioblastoma samples with radiotherapy or TMZ/radiotherapy, age 27-70 years), GSE10878 (origin: normal tissue and primary glioblastoma tissue, age: 39-76 years), GSE46016 (origin: neural stem cells and human glioblastoma stem cells, age: $33-71$ years), GSE15824 (origin: normal brain tissue \& astrocytes and primary glioblastoma tissue, age: 35-70 years), GSE31262 (origin: non-tumoral brain tissue and primary glioblastoma tissue, age:33-71 years), GSE42656 (origin: adult control cerebellum and pediatrics glioblastoma, age:182 weeks), and GSE50161 (origin: normal brain samples and primary tumor (glioblastoma), age: 35-70 years) were analyzed. Other types of gliomas such as astrocytoma, oligodendroglioma, medulloblastoma were not considered for the analysis. The data was $\log 2$ transformed and then a differential analysis was performed using standard Limma function ( $\mathrm{R}$ package for the analysis of differential gene expression [16]). The normalized data further scaled between 0 and 1 using formula $\mathrm{Zi}=\mathrm{xi}-\min (\mathrm{x}) / \max (\mathrm{x})-$ $\min (x)$, where $x$ represent the expression.

\section{Protein-protein interaction network}

Information on proteins that interact with ETBR was obtained with a protein neighborhood analysis tool [17]. The interacting partners were shown with Cytoscape, an online open source software tool to display molecular interaction networks and biological pathways [18]. Gene ontology of ETBR was obtained with a gene set enrichment tool and enrichment scores as described [19].

\section{Proof-of-concept: in vitro cytotoxic assays}

To test whether endothelin receptor blockers affect GBM cell growth and toxicity in vitro, three drugs currently in use to treat pulmonary artery hypertension were used-ambrisentan (Letairis/Volibris), which selectively blocks the ETAR, and macitentan (Opsumit) and bosentan (Tracleer), which block both ETAR and ETBR-in standard viability assays with a CellTiter 96 Aqueous One Solution Cell Proliferation Assay system (Promega) as recommended by the manufacturer. We also tested BQ788, which is selective for ETBR, and ACT-132577, the active metabolite of macitentan. The ACT-132577, bosen$\tan$ BQ788 and macitentan used in this study were from Medchemexpress LCC (Princeton, NJ, USA), while ambrisentan was from Ark Pharm, Inc. (Libertyville, IL, USA). All the drugs were provided and checked by Medivir, Stockholm, Sweden. To test the effects of the drugs on cancer cells, we used primary GBM cells (GBM30, GBM42, GBM48, GBM392, and GBM398) and three GBM lines (U-251 MG, U-373 MG (Uppsala), and U-343 MGa). To test drug effects on normal cells, we used human umbilical vein endothelial cells (HUVECs, Lonza, CH-3930 Visp, Switzerland), MRC-5 lung fibroblasts (ATCC, LGC Standards, Middlesex, UK), and retinal pigment epithelial cells (a generous gift from Dr. Rich Stanton, Cardiff University). Since the endothelin axis (consisting of endothelins, ETAR, and ETBR) has also been implicated in breast cancer, we also tested the drugs on three breast cancer lines: MCF7, MDA-MA-231, and SK-BR-3. In brief, approximately $1 \times 10^{4}$ cells/well were seeded onto 96-well plates and treated with twofold serial dilutions of drug $(0.78-200 \mu \mathrm{M})$. Cell viability was assessed on day 6 with a VersaMax ELISA Microplate Reader (Molecular Devices, Wokingham, Berkshire, UK) at an optical density of $490 \mathrm{~nm}$ (reference wave length, $650 \mathrm{~nm}$ ). The optical density of treated cells was expressed as a percentage of untreated cells, which were considered $100 \%$ viable. 


\section{Statistical analysis}

$P<0.05$ was considered to indicate statistical significance. Survival curves were estimated with the KaplanMeier method, and the significance of differences between the curves was determined with the log-rank test. Boxplots were plotted with $\mathrm{R}$ programming and analyzed by $t$ test.

\section{Results}

\section{Expression data from TCGA and GEO database}

To determine whether ETBR is overexpressed in GBM, we analyzed the ETBR mRNA expression in TCGA and GEO databases. In TCGA, mRNA expression data $(n=$ 171) demonstrate that the median expression of ETBR was significantly higher in primary (de novo) GBM tumors than in normal aging control brains (Fig. 1a). Similarly, ETBR mRNA expression was higher in patients with untreated primary (de novo) GBM tumor than in tumors from patients treated for primary GBM (from the Affymetrix HuExGeneChip mRNA microarray data, quantile normalized; $n=517$ ) (Fig. 1b). Interestingly, patients with untreated primary (de novo) GBM tumors with higher median expression of ETBR tended to have shorter survival compared to those with lower median expression (Fig. 1c). The survival data from GSE7696 and GSE16011 cohorts also showed that patients with over-median expression of ETBR had lower survival rates at 3 years than 5 years, respectively (Additional file 1: Figure S1). In the GEO datasets GSE2223, GSE7696, GSE10878, GSE46016, GSE15824, and GSE31262, ETBR expression was significantly higher in GBM patients than in controls (Fig. 2); no difference of ETBR expression was noted in datasets GSE42656, and GSE50161 (Additional file 1: Figure S2), perhaps mirroring the heterogeneity of the disease.
To further investigate the expression levels of ETBR in other cancers, we analyzed ETBR expression in silico with available datasets. ETBR expression varied among cancer types but was higher in malignant cancer, mixed glioma, GBM, and melanoma (Fig. 3 and Additional file 1: Figure S3). Taken together, these data predicted that overexpression of ETBR may be a prognostic marker for a subset of GBM patients and potentially for other tumor types as well (Additional file 1: Figure S3).

\section{ETBR is overexpressed in GBM}

To confirm the higher expression of ETBR shown by the bioinformatics analysis in tissue specimens, we examined tumor tissue specimens obtained from 25 GBM patients we studied in a previous cohort [15] fro ETBR expression. ETBR expression was low in $64 \%(n=16)$ and high in $36 \%(n=9)$. ETBR was predominantly detected in the cytoplasm of tumor cells and was not found in adjacent nontumor cells, consistent with previous findings [20]. Little or no ETBR immunoreactivity was detected in control brains $(n=10)$ (Fig. 4) and was mainly located in corpora amylacea and occasionally in some arteriole-like structures.

\section{Overexpression of ETBR is correlated with a shorter overall survival}

To determine whether ETBR expression levels are of prognostic value for GBM patients, we used the KaplanMeier method to analyze the survival of 25 patients with low or high ETBR expression levels. ETBR expression correlated inversely and significantly with the survival times of these GBM patients (Fig. 5). Interestingly, we observed higher expression of ETBR in both 'Classical' and 'Neural' subtypes of GBM according to molecular classification [21] that tended to be correlated with poor overall survival (Additional file 1: Figure S5A-B).
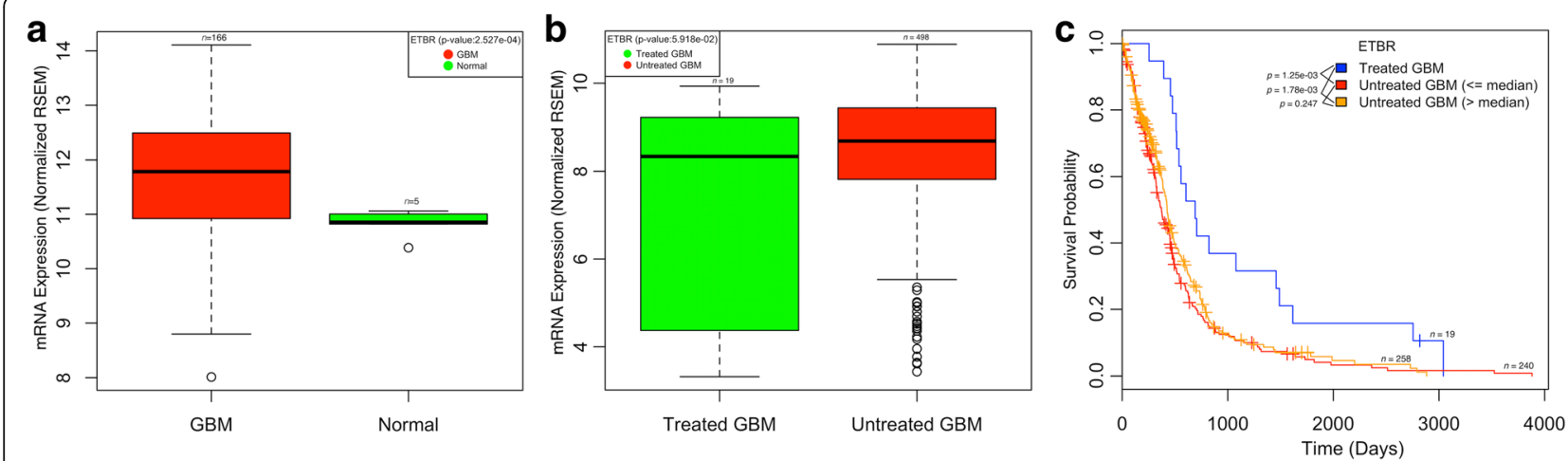

Fig. 1 Endothelin receptor type B (ETBR) mRNA expression and its correlation with GBM patient's survival as determined by bioinformatics analysis of the TCGA database. a ETBR mRNA expression was significantly higher in patients with GBM $(n=166)$ than in normal controls $(n=5)$ as normalized with RSEM (RNA-Seq by Expectation Maximization) software [35] b ETBR expression was higher in patients with untreated GBM than in those with treated GBM. c Survival curves based on clinical information and ETBR mRNA expression of treated and untreated GBM reported in the TCGA database 

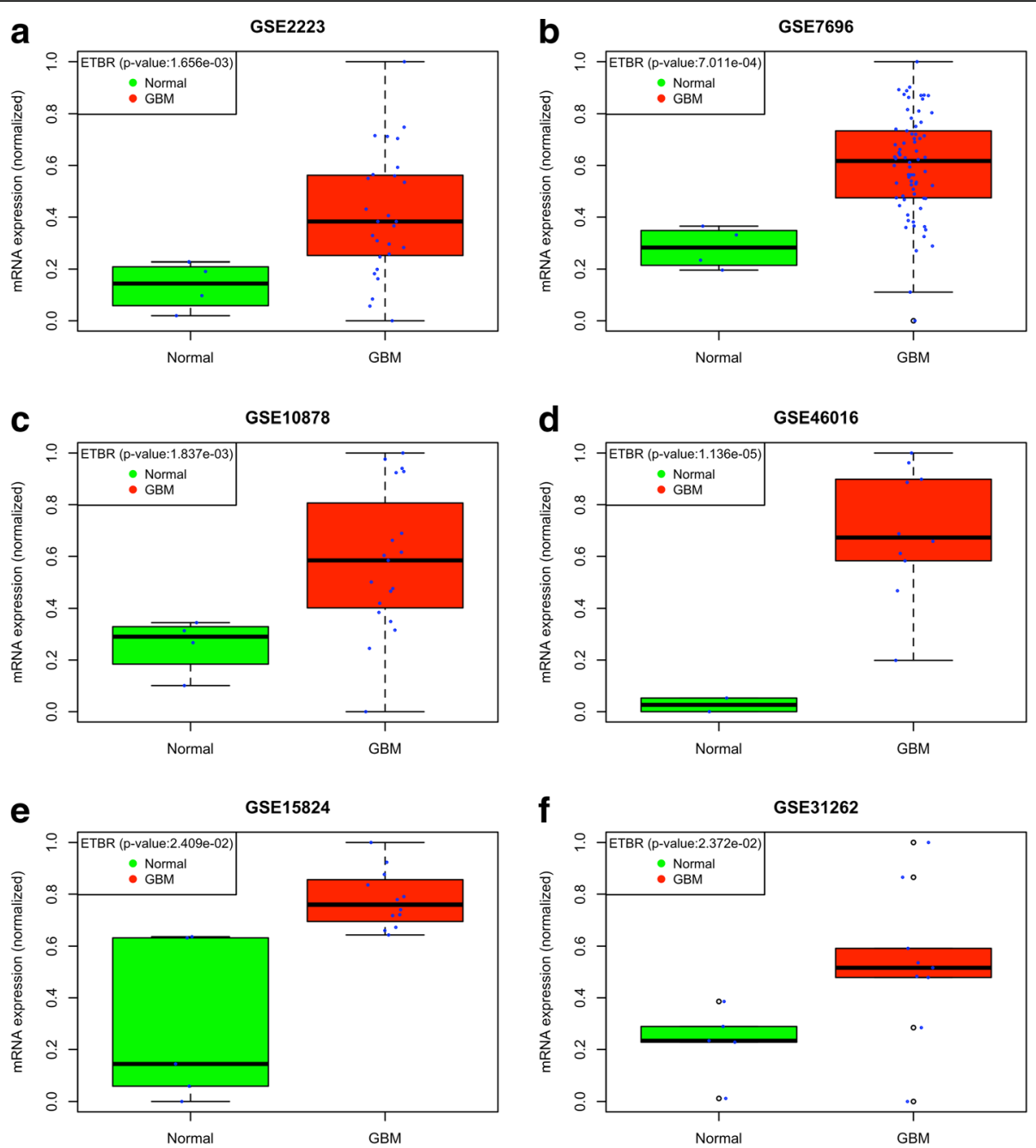

Fig. 2 Normalized ETBR mRNA expression determined by analyzing GEO datasets (a-f). ETBR mRNA expression is shown for control (normal) and GBM patients. The signifance obtained from t-test is shown in inset box

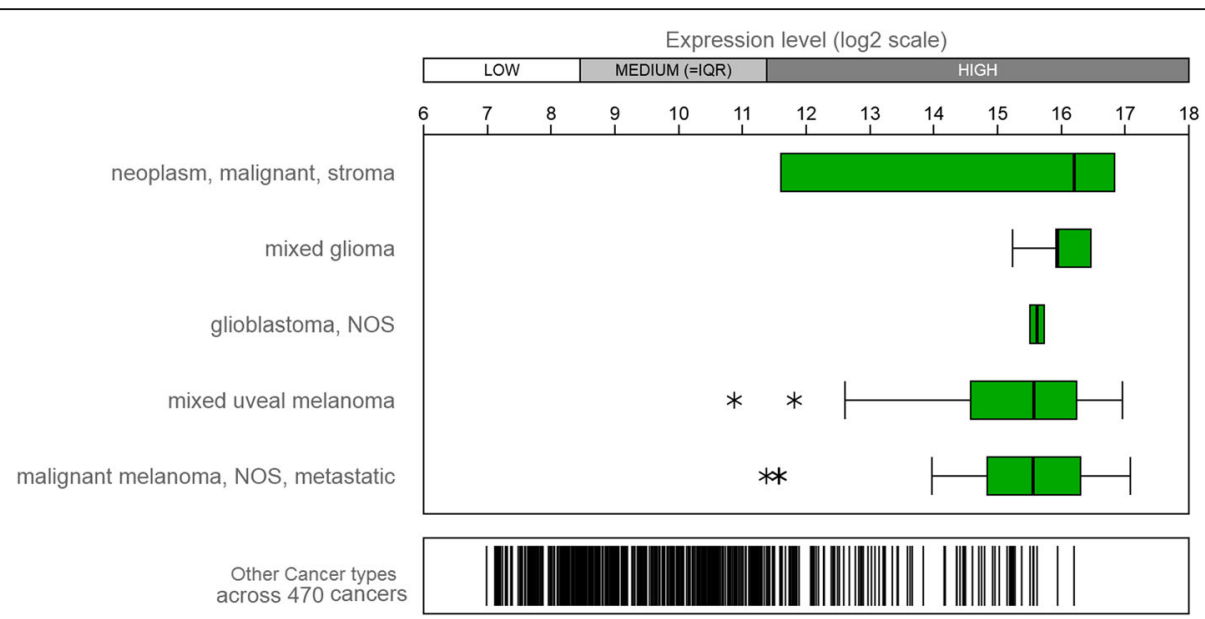

Fig. 3 ETBR expression level in different cancers. The expression level of ETBR in human cancers is shown from Affymetrix Human Genome U133 Plus 2.0 Array. Enrichment of five major cancers in ETBR expression, shown with Genevestigator (http://genevestigator.com/gv/). NOS = Not Otherwise Specified, accordingly to WHO classification of CNS tumors (2016) 

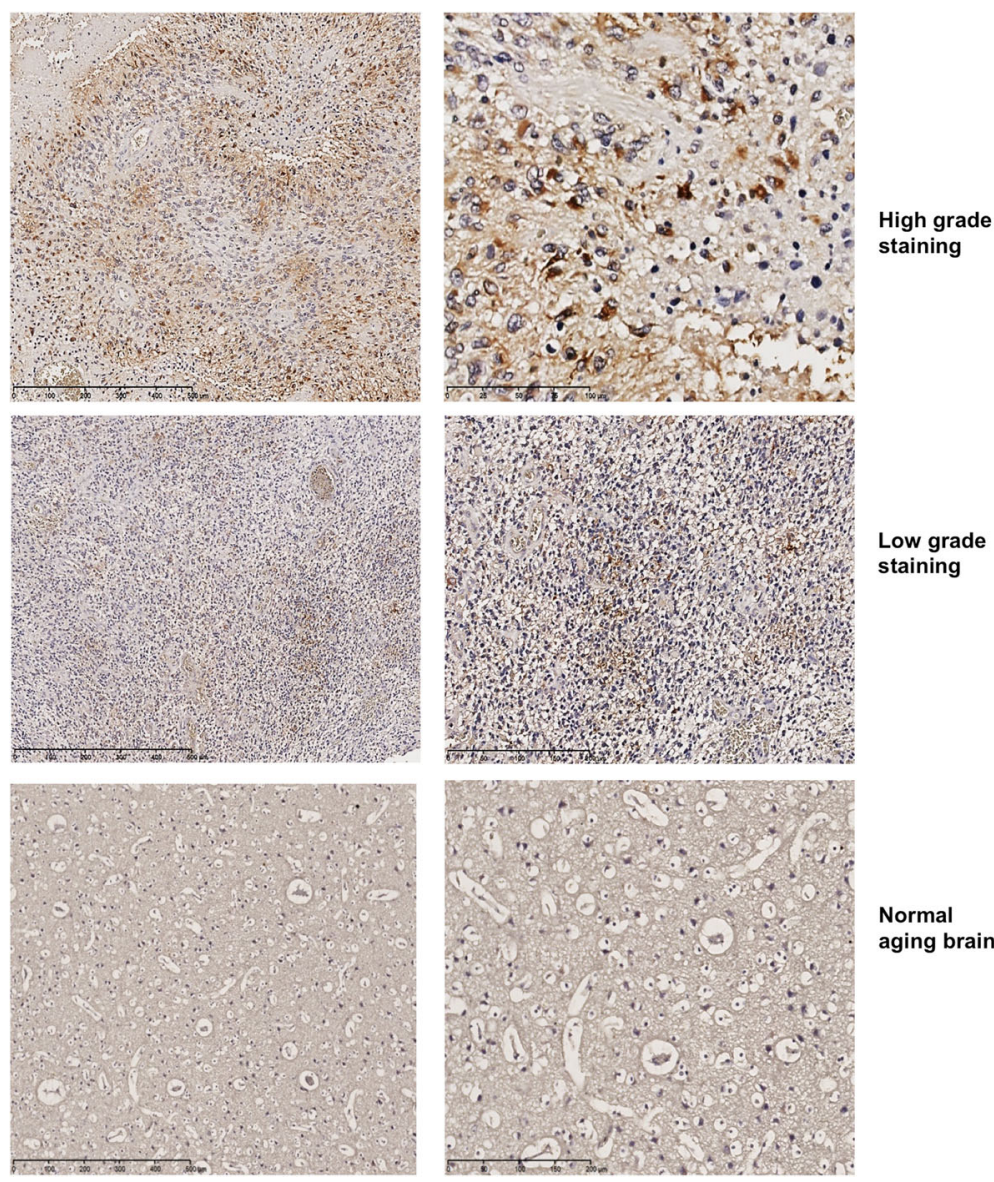

Fig. 4 Protein expression of ETBR in GBM by immunohistochemistry staining. Representative photomicrographs showing high or low grade of ETBR staining (brown). Normal aging brains (frontal part) served as controls. Right panel is a higher magnification of the left panel

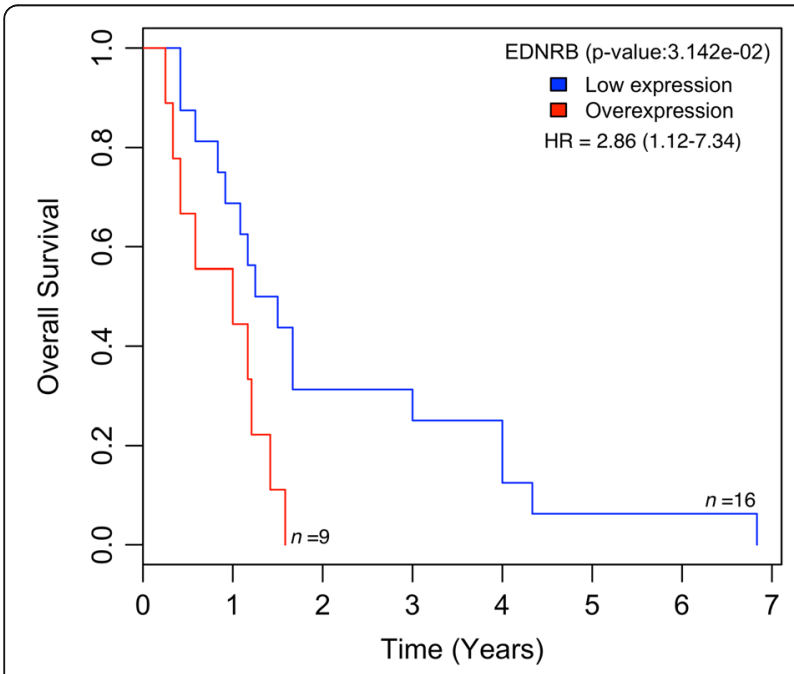

Fig. 5 Kaplan-Meier survival curve for ETBR in 25 Swedish GBM patients. Overexpression of ETBR is correlated with shorter survival, whereas lower expression of ETBR is correlated with longer survival

\section{Druggability of ETBR}

Next, we used network neighborhood analysis to examine possible interaction networks and signaling pathways of ETBR. The general structure of ETBR is shown in Fig. 6a. ETBR is known to be expressed on the plasma membrane, in the cytosol and on the nuclear membrane (Fig. 6b). The analysis showed that ETBR potentially interacts with eight proteins: guanine nucleotide-binding protein subunit alpha-11, guanine nucleotide-binding protein subunit alpha-13, caveolin-1, G protein-coupled receptor kinase 6, endothelin-1, endothelin-3, adrenergic beta receptor kinase 1 , and nitric oxide synthase 3 (Fig. 6c). Further expansion of protein neighbors showed 175 interacting partners, many of which are involved in cell-cell communications (gap junction, adherens junction), the vascular endothelial growth factor signaling pathway, and calcium signaling that is associated with cancer pathogenesis (Fig. 6d). In addition, the ETBR-interacting proteins (up to second neighbor) acted as signature genes in different cancers; 11 proteins were observed in melanoma, 10 proteins in lung and stomach adenocarcinoma, and 5 proteins in GBM (Additional file 


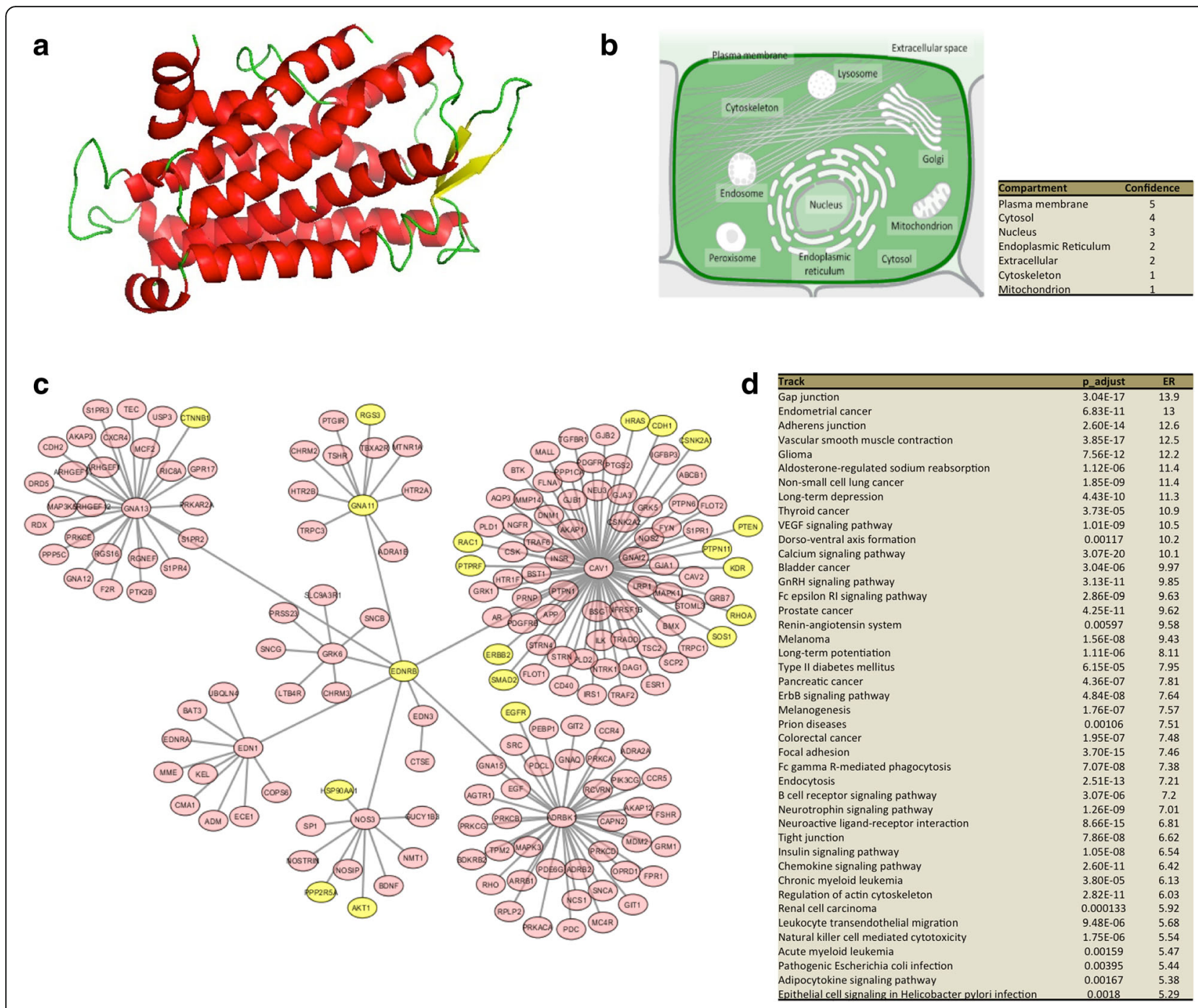

Fig. 6 In silico analysis of the protein structure and cellular localization of ETBR protein and its protein-protein interactions. A) The SWISS model of ETBR protein (transmembrane, G protein-coupled receptor), residues 111-398. Helices are shown in red, sheets in yellow, and loops in green. B) ETBR is primarily localized to the plasma membrane, cytosol, and nuclear membrane as revealed by COMPARTMENTS resource (http://compartments.jensenlab.org). C) Analysis of protein-protein interactions shows that ETBR primarily interact with eight proteins, which interact with 175 proteins. D) Gene ontology of ETBR neighborhood proteins

1: Figure S4A) [22]. We also found an association between cancer types and ETBR-interacting proteins (Additional file 1: Figure S4B). Collectively, these data suggest that ETBR is a potential therapeutic target in GBM and other cancers.

\section{In vitro viability assay}

To substantiate our prediction that ETBR is a druggable target for GBM and other cancer types, we used a standard viability assay to assess the cytotoxic effects of three clinically available endothelin receptor blockers-macitentan, bosentan, and ambrisentan-on primary GBM cells and GBM and breast cancer cell lines as compared with normal fibroblasts, endothelial and epithelial cells. Macitentan and its active metabolite (ACT-132577) reduced the viability of all GBM primary cells and cell lines tested; the effects were dose dependent (Fig. 7a-h). Bosentan had similar dose-dependent effects (Fig. 7a-c, $\mathrm{f}-\mathrm{h})$. In contrast, ambrisentan was not cytotoxic, even at the highest tested dose (Fig. $7 \mathrm{~d}-\mathrm{h}$ ), apart from a minor trend toward reduced viability of GBM398 (Fig. 7e). Strikingly, at the highest dose, BQ788 dramatically reduced the viability of primary GBM392 cells and cell line U-343 MGa (Fig. 7a-h). Similar dose-dependent effects in breast cancer cells lines were observed for bosentan and for macitentan and its active metabolite ACT132577; ambrisentan was not cytotoxic, and BQ788 had a single dramatic effect (Fig. 8a-c). Normal fibroblasts and epithelial cells tolerated ambrisentan and BQ788 well at $100 \mu \mathrm{M}$ but not at $200 \mu \mathrm{M}$ (Fig. 8d and e, 

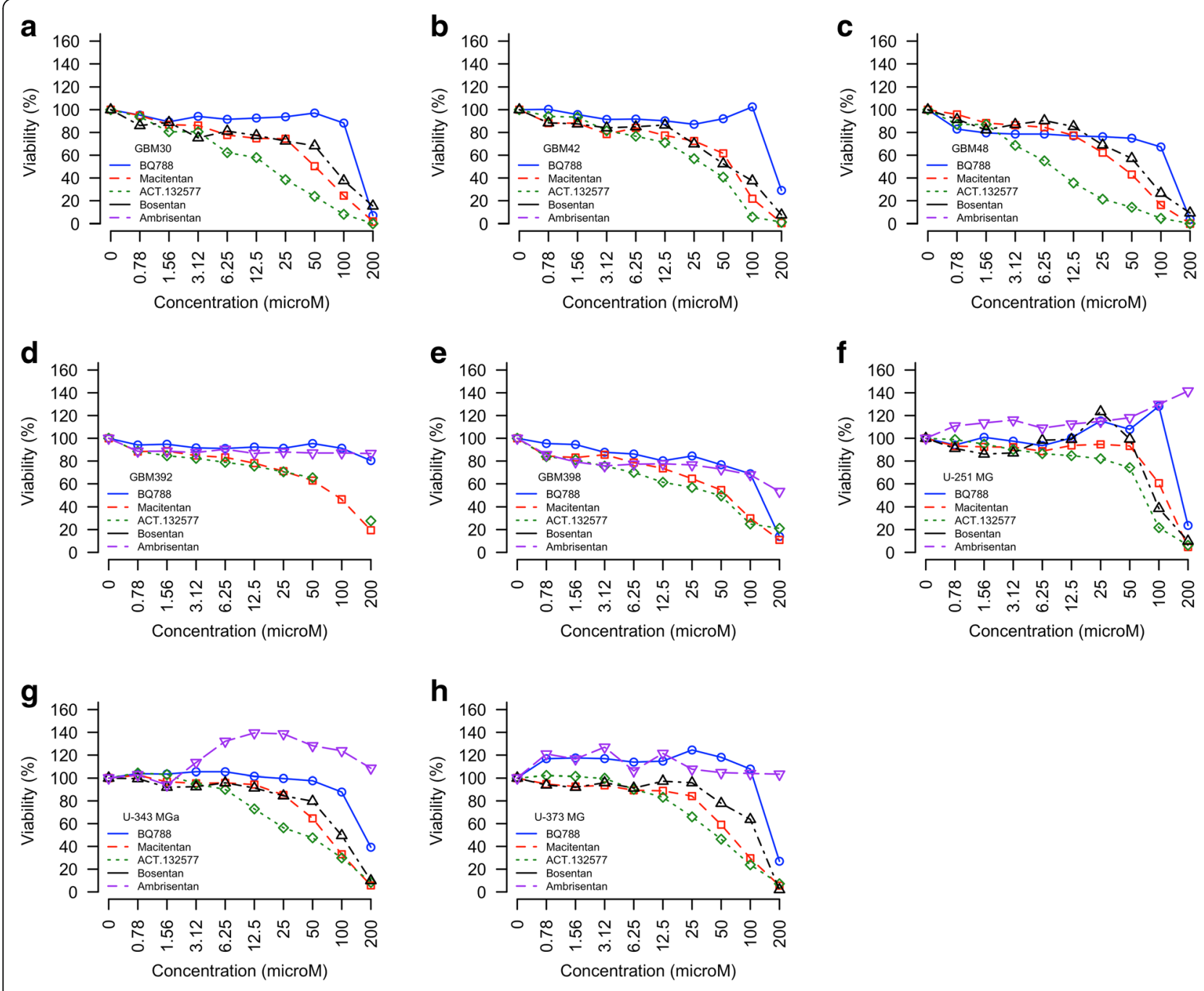

Fig. 7 The effects of endothelin receptor antagonists on GBM. a-e Primary cells $(n=3)$. $\mathbf{f}-\mathbf{h}$ Cell lines $(n=6)$

respectively). Macitentan and its active metabolite resulted in $40-50 \%$ cell death at $100 \mu \mathrm{M}$ in both cell types; HUVECs were more sensitive to macitentan and its metabolite (Fig. 8f). Notably, there is a differential baseline expression of ETBR in primary GBM tissues/cells and breast cancer lines compared to that of normal fibroblasts, endothelial and epithelial cells (Additional file 1: Figure S6). These findings suggest the potential feasibility of using endothelin receptor antagonists to treat GBM and breast cancer, and that fine-tuning of the ETAR and ETBR balance is crucial for maximum cytotoxicity while sparing normal cells.

\section{Discussion}

In this study, we investigated whether ETBR is overexpressed in GBM tumors in a Swedish patient cohort and assessed the potential usefulness of ETBR as a prognostic marker and drug target for GBMs and other types of cancer. We found that ETBR is indeed often overexpressed in GBM tumors, with little or no immunoreactivity in control brains. Analysis of expression data from TCGA and a subset of GEO datasets showed that overexpression of ETBR in GBM was correlated with shorter patient survival. Similarly, by examining ETBR expression across 470 cancers, glioma or GBM were again found to have high expression. By mapping the protein neighborhood to ETBR, we found that ETBR is mainly predicted to interact with eight proteins that further interact with 175 additional proteins, many of which are involved in cell-cell communication (gap junction, adherens junction), the vascular endothelial growth factor signaling pathway, and calcium signaling-all of which are associated with cancer pathogenesis. These results support the potential use of ETBR blockers as a targeted therapy for cancer [10].

The endothelin axis has been implicated in the pathogenesis of many types of cancers (reviewed in [23]). In 


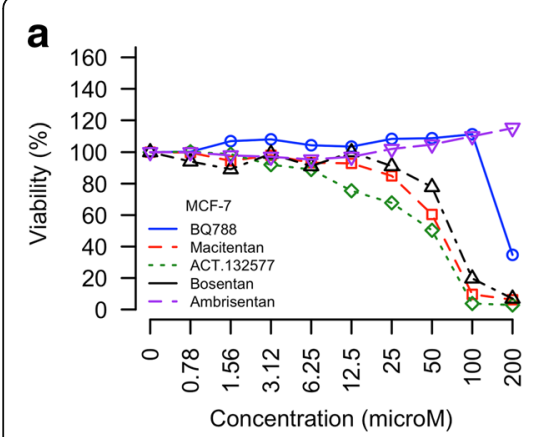

d

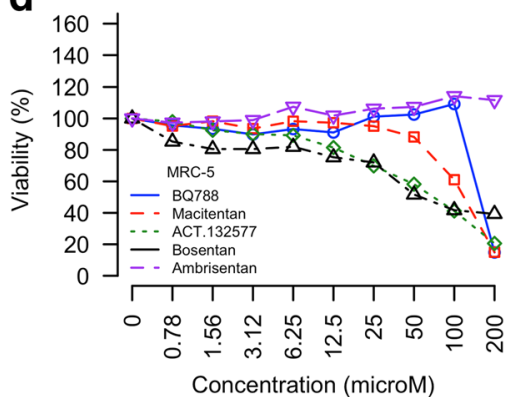

b

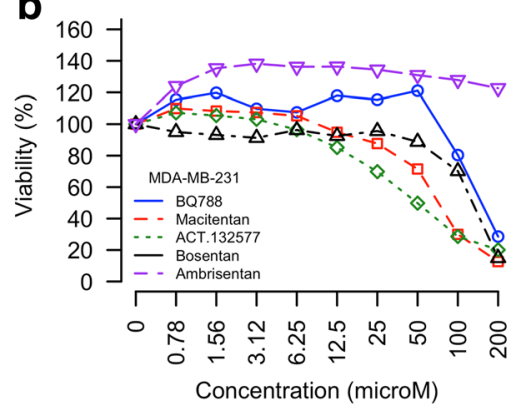

e

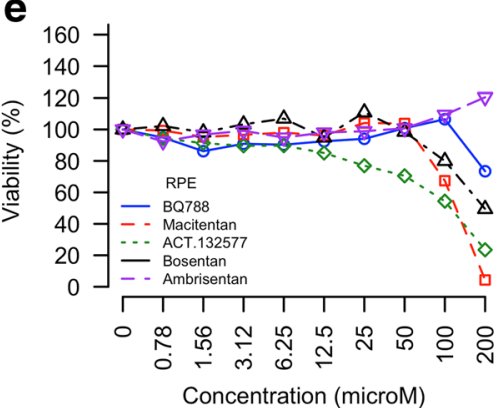

C

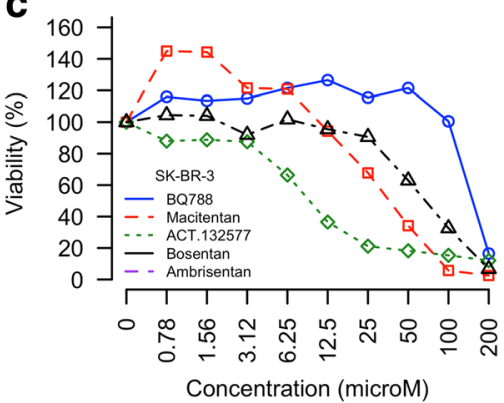

f

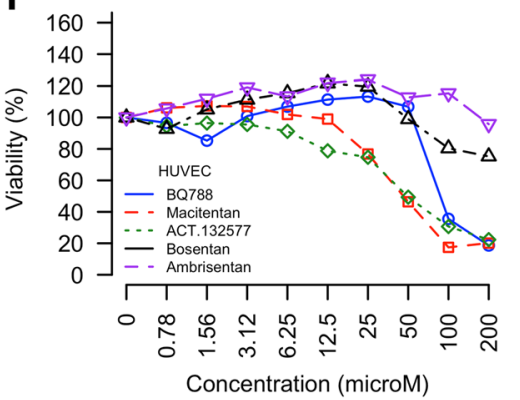

Fig. 8 The effects of endothelin receptor antagonists on breast cancer cells and normal cells. a-c Breast cancer cell lines ( $n=6$ for MCF-7 and MDA-MB-231; $n=3$ for SK-BR-3). $\mathbf{d}-\mathbf{f})$ Normal cells: MRC-5 fibroblasts $(\mathbf{d}, n=6)$, retinal pigment epithelial cells (RPE) $(\mathbf{e}, n=6)$, and human umbilical vein endothelial cells (HUVEC) $(\mathbf{f}, n=6)$

particular, ETBR is overexpresssed in bladder carcinoma [24], melanoma [25], small-cell lung cancer [26], vulvar cancer [5], clear-cell renal cell carcinoma [6], oesophageal squamous cell carcinoma [7], and astrocytoma (including GBM) [12]. ETBR was also earlier reported to be highly expressed in melanoma [25]. Of note, ETBR overexpression was correlated with shorter patient survival or poor patient outcome in small-cell lung cancer, vulvar cancer, clear-cell renal cell carcinoma, esophageal squamous cell carcinoma, and GBM [5-7, 12, 24, 27] and may thereby represent a potential prognostic marker as well as a therapeutic target for several cancer forms. We confirmed this hypothesis in the current study. We assessed the toxicity of ETBR and ETAR blockers for cancer cells of different origins. While Ambrisentan was not cytotoxic to GBM cells or breast cancer cells, the ETBR-selective blocker BQ788, the dual ETBR and ETAR blockers bosentan and macitentan, and the active metabolite of macitentan, ACT132577 inhibited tumor cell growth to some extent. The affinity of ambrisentan to ETBR is at most $1 \%$ of its affinity to ETAR $\left(\mathrm{IC}_{50}=1 \mathrm{nM}\right)$ (reviewed in [28]), and ambrisentan was the least effective of the drugs we tested. We speculate that the ratio between ETAR and ETBR may be crucial for maximum cytotoxic efficiency.

To our knowledge, only two studies have earlier demonstrated overexpression of ETBR in GBM tumors, one study of Han-Chinese patients [12] and one in Japanese patients [29]. Ethnicity is a factor in the pathogenesis of gliomas $[13,14]$. Epidemiological data suggest that the incidence of glioma in the United States is higher among whites, followed by blacks, Hawaiians, Chinese or Japanese, Filipinos, and Alaskan natives [30]. The incidence of gliomas is also higher in Scandinavian countries than in Asian countries [30]. The ethnicity difference may be related to or result in alterations in the expression of key proteins. The promoter methylation status of the $O^{6}$ methylguanine methyltransferase, a key DNA repair enzyme predicted the outcome of treatments that include an alkylating agent in Caucasian populations but not in Indians [31]. In the present study, we detected higher ETBR immunoreactivity in tumor cells from GBMs of Swedish patients, while little or no ETBR immunoreactivity was detected in adjacent nontumor cells, which consistent with a previous report [20]. ETBR is known to predominantly express by astrocytes, where it helps regulate cell hypertrophy [32]. In normal aging brains, we detected ETBR immunoreactivity in corpora amylacea, which are glycoproteinaceous inclusion bodies associated with aging or neurodegenerative diseases. The significance of this finding is unknown but it is well-known that the corpora amylacea is immunoreactive to various proteins (reviewed in [33]) and recently, to an antibody against a late antigen (MAB8127, Millipore) of human cytomegalovirus, a ubiquitous beta herpesvirus [34].

Our study is limited by the relatively small number of patients. Hence a large-scale patient cohort is needed to 
further evaluate the usefulness of ETBR as prognostic marker. A future study should also be tailored to better understand the role of ETBR in the pathogenesis of GBM. Nevertheless, our study confirms that ETBR is overexpressed in GBM and other cancer forms and further implicates ETBR as a potentially useful prognostic marker and possibly a therapeutic target for cancer.

\section{Conclusion}

This study examined the potential role of ETBR in GBM tumors as well as in other cancer forms. ETBR expression was higher in GBM tumors and several other cancer forms than in control tissues and high ETBR expression was correlated with poor patient outcome. ETBR blockers were in general more toxic to tumor cells than normal cells, which imply a potential benefit of ETBR blockers in cancer therapy.

\section{Additional file}

Additional file 1: Figure S1. Survival curves based on clinical information obtained from GSE7696 and GSE16011 for Endothelin receptor type B (ETBR) mRNA expression in GBM. GSE7696 and GSE16011 cohort shows that over-median expression of ETBR has lower survival rate at 3 years than 5 years (http://watson.compbio.iupui.edu/). Figure S2. ETBR mRNA expression in GSE42656 (A) and GSE50161 (B). No statistically significant difference was observed for ETBR expression in normal brain tissue compared to that of GBM patients, mirroring heterogeneity of disease. Figure S3. Overexpression of ETBR mRNA in different cancers of the TCGA cancer cohort is shown using The Cancer Cell Line Encyclopedia (CCLE, https:/portals.broadinstitute.org/ccle). Numbers in parentheses indicate sample size. Figure S4. The ETBR-interacting proteins (up to second neighbor) were searched in different cancer signature genes as described [22] A). The number of ETBR-interacting proteins found to be signature genes in various cancer cohorts were shown. B) The association between cancer types and ETBR-interacting proteins suggests a possible role of ETBR in GBM and other cancers. Cancer types are shown with degree-based node shape and surrounding red circles, whereas ETBR-interacting proteins are shown with degree based nodes (orange). Figure S5. ETBR expression in different subtypes of GBM (A) and its correlation with survival (B) according to molecular classification [21]. Higher expression of ETBR in both 'Classical' and 'Neural' subtype tended to be correlated with poor overall survival. Figure S6. Relative expression of ETBR in various primary GBM cells, breast cancer lines (MCF-7, SKBR3 and MDA-MB-231), fibroblast (MRC-5), endothelial cells (HUVEC) and epithelial cells (RPE) as normalized to MRC-5. (DOC 2199 kb)

\section{Abbreviations}

ERA: Endothelin receptor antagonists; ETAR: Endothelin A receptor: ETBR: Endothelin B receptor; GBM: Glioblastoma

\section{Acknowledgements}

We thank all lab members for valuable comments and Stephen Ordway for editorial assistance; Drs. Anna Martinez Casals and Mohsen Karimi Arzenani for the RNA and CDNA derived from GBM tissues, respectively; Anna Ridderstad Wollberg from VINNOVA-BIO-X for excellent coaching; Ylva Terelius, Richard Bethell, Fredrik Öberg and Susana Ayesa Alvarez from Medivir AB for useful technical advice and helped with the procurement and purity analysis of the endothelin antagonists.

\section{Funding}

This work was supported by Sten A Olssons Foundation for Research and Culture, Family Erling-Persson Foundation, Torsten Söderberg Foundation, BILTEMA Foundation, IngaBritt and Arne Lundbergs Foundation, Stichting af Jochnick Foundation, Jane and Dan Olssons Research Foundation, Nexttobe,
Swedish Cancer Foundation, Children's Cancer Foundation, Swedish Medical Research Council, Thematic Cardiovascular Research Center and Stockholm County Council, and the Swedish Heart-Lung Foundation, VINNOVA-BIO-X and Medivir AB (all to CSN), Cure cancer /Bota Cancer (to KCY). The funding body had no role in the design of the study and collection, analysis, and interpretation of data and in writing the manuscript.

\section{Availability of data and materials}

All data generated or analysed during this study are included in this published article [and its Additional files].

\section{Authors' contributions}

KCY, GT and SV conceived and designed the study; SV, GT, NL, HC, HLC, BD and KCY did the research; VW, AAM, PS, SA, MS, KS, GS, AR, KTW and JT contributed new reagents or analytic tools; SV, GT, CSN and KCY analyzed the data and wrote the paper. All authors read and approved the final manuscript.

\section{Ethics approval and consent to participate}

The use of patient materials was approved by the Ethics Committee at the Karolinska Institutet and by the Medical Ethics Committee, University of Malaya Medical Center, Malaysia, and conducted in accordance with the Declaration of Helsinki.

\section{Consent for publication}

Not applicable.

\section{Competing interests}

A patent application for use of ETBR inhibitors in prevention and treatment of HCMV infection and CMV-related pathologies such as cardiovascular diseases and cancer has been filed (to CSN and KCY).

\section{Publisher's Note}

Springer Nature remains neutral with regard to jurisdictional claims in published maps and institutional affiliations.

\section{Author details}

${ }^{1}$ Unit of Computational Medicine, Center for Molecular Medicine, Department of Medicine, Karolinska Institutet, Stockholm, Sweden. ${ }^{2}$ Cell and Molecular Immunology, Experimental Cardiovascular Unit, Departments of Medicine and Neurology, Center for Molecular Medicine, Karolinska Institutet, SE-171 76 Stockholm, Sweden. ${ }^{3}$ Department of Cell and Molecular Biology, Karolinska Institutet, Stockholm, Sweden. ${ }^{4}$ Department of Neurosurgery, Karolinska University Hospital, Stockholm, Sweden. ${ }^{5}$ Department of Pathology, University of Malaya, Kuala Lumpur, Malaysia. ${ }^{6}$ Biological and Environmental Sciences and Engineering Division (BESE), Computer, Electrical and Mathematical Sciences and Engineering Division (CEMSE), King Abdullah University of Science and Technology (KAUST), Thuwal 23955-6900, Kingdom of Saudi Arabia.

Received: 30 December 2016 Accepted: 22 January 2018 Published online: 06 February 2018

\section{References}

1. Ohgaki H, Kleihues P. Population-based studies on incidence, survival rates, and genetic alterations in astrocytic and oligodendroglial gliomas. J Neuropathol Exp Neurol. 2005;64(6):479-89.

2. Wen PY, Kesari S. Malignant gliomas in adults. N Engl J Med. 2008;359(5): 492-507.

3. Gruber ML, Buster WP. Temozolomide in combination with irinotecan for treatment of recurrent malignant glioma. Am J Clin Oncol. 2004;27(1):33-8.

4. Mazzuca MQ, Khalil RA. Vascular endothelin receptor type B: structure, function and dysregulation in vascular disease. Biochem Pharmacol. 2012; 84(2):147-62.

5. Eltze E, Bertolin M, Korsching E, Wulfing P, Maggino T, Lelle R. Expression and prognostic relevance of endothelin-B receptor in vulvar cancer. Oncol Rep. 2007;18(2):305-11.

6. Wuttig D, Zastrow S, Fussel S, Toma MI, Meinhardt M, Kalman K, Junker K, Sanjmyatav J, Boll K, Hackermuller J, et al. CD31, EDNRB and TSPAN7 are promising prognostic markers in clear-cell renal cell carcinoma revealed by 
genome-wide expression analyses of primary tumors and metastases. Int Cancer. 2012;131(5):E693-704.

7. Tanaka T, Sho M, Takayama T, Wakatsuki K, Matsumoto S, Migita K, Ito M, Hamada K, Nakajima Y. Endothelin B receptor expression correlates with tumour angiogenesis and prognosis in oesophageal squamous cell carcinoma. Br J Cancer. 2014;110(4):1027-33.

8. Bagnato A, Loizidou M, Pflug BR, Curwen J, Growcott J. Role of the endothelin axis and its antagonists in the treatment of cancer. Brit $J$ Pharmacol. 2011;163(2):220-33

9. Wan $X$, Zhang LB, Jiang B. Role of endothelin B receptor in oligodendroglioma proliferation and survival: in vitro and in vivo evidence. Mol Med Rep. 2014;9(1):229-34.

10. Asundi J, Reed C, Arca J, McCutcheon K, Ferrando R, Clark S, Luis E, Tien J, Firestein $R$, Polakis P. An antibody-drug conjugate targeting the endothelin B receptor for the treatment of melanoma. Clin Cancer Res. 2011;17(5):965-75.

11. Kim SJ, Lee HJ, Kim MS, Choi HJ, He J, Wu Q, Aldape K, Weinberg JS, Yung WK, Conrad CA, et al. Macitentan, a dual Endothelin receptor antagonist, in combination with Temozolomide leads to Glioblastoma regression and long-term survival in mice. Clin Cancer Res. 2015;21(20):4630-41.

12. Shen $C$, Yang $L$, Yuan $X$. Endothelin B receptor expression in human astrocytoma: association with clinicopathological variables and surviva outcomes. Int J Neurosci. 2011;121(11):626-31.

13. Ratneswaren $T$, Jack RM, Tataru D, Davies EA. The survival of patients with high grade glioma from different ethnic groups in south East England. J Neuro-Oncol. 2014;120(3):531-6.

14. Chen P, Aldape K, Wiencke JK, Kelsey KT, Miike R, Davis RL, Liu J, Kesler-Diaz A, Takahashi M, Wrensch M. Ethnicity delineates different genetic pathways in malignant glioma. Cancer Res. 2001;61(10):3949-54.

15. Rahbar A, Orrego A, Peredo I, Dzabic M, Wolmer-Solberg N, Straat K, Stragliotto G, Soderberg-Naucler C. Human cytomegalovirus infection levels in glioblastoma multiforme are of prognostic value for survival. J Clin Virol. 2013:57(1):36-42.

16. Ritchie ME, Phipson B, Wu D, Hu Y, Law CW, Shi W, Smyth GK. limma powers differential expression analyses for RNA-sequencing and microarray studies. Nucleic Acids Res. 2015;43(7):e47.

17. Vasaikar SV, Padhi AK, Jayaram B, Gomes J. NeuroDNet - an open source platform for constructing and analyzing neurodegenerative disease networks. BMC Neurosci. 2013;14:3.

18. Smoot ME, Ono K, Ruscheinski J, Wang PL, Ideker T. Cytoscape 2.8: new features for data integration and network visualization. Bioinformatics. 2011;27(3):431-2.

19. Subramanian A, Tamayo P, Mootha VK, Mukherjee S, Ebert BL, Gillette MA, Paulovich A, Pomeroy SL, Golub TR, Lander ES, et al. Gene set enrichment analysis: a knowledge-based approach for interpreting genome-wide expression profiles. P Natl Acad Sci USA. 2005;102(43):15545-50.

20. Egidy G, Eberl LP, Valdenaire O, Irmler M, Majdi R, Diserens AC, Fontana A, Janzer RC, Pinet F, Juillerat-Jeanneret L. The endothelin system in human glioblastoma. Lab Investig. 2000;80(11):1681-9.

21. Verhaak RG, Hoadley KA, Purdom E, Wang V, Qi Y, Wilkerson MD, Miller CR Ding $L$, Golub T, Mesirov JP, et al. Integrated genomic analysis identifies clinically relevant subtypes of glioblastoma characterized by abnormalities in PDGFRA, IDH1, EGFR, and NF1. Cancer Cell. 2010;17(1):98-110.

22. Rubio-Perez C, Tamborero D, Schroeder MP, Antolin AA, Deu-Pons J, PerezLlamas C, Mestres J, Gonzalez-Perez A, Lopez-Bigas N. In silico prescription of anticancer drugs to cohorts of 28 tumor types reveals targeting opportunities. Cancer Cell. 2015;27(3):382-96.

23. Bagnato A, Spinella F, Rosano L. The endothelin axis in cancer: the promise and the challenges of molecularly targeted therapy. Can J Physiol Pharm. 2008;86(8):473-84.

24. Eltze E, Wild PJ, Wulfing C, Zwarthoff EC, Burger M, Stoehr R, Korsching E, Hartmann A. Expression of the endothelin axis in noninvasive and superficially invasive bladder cancer: relation to clinicopathologic and molecular prognostic parameters. Eur Urol. 2009;56(5):837-45.

25. Demunter A, De Wolf-Peeters C, Degreef H, Stas M, van den Oord JJ. Expression of the endothelin-B receptor in pigment cell lesions of the skin evidence for its role as tumor progression marker in malignant melanoma. Virchows Arch. 2001;438(5):485-91.

26. Blouquit-Laye S, Regnier A, Beauchet A, Zimmermann U, Devillier P, Chinet T. Expression of endothelin receptor subtypes in bronchial tumors. Oncol Rep. 2010;23(2):457-63.
27. de Tayrac M, Aubry M, Saikali S, Etcheverry A, Surbled C, Guenot F, Galibert MD, Hamlat A, Lesimple T, Quillien V, et al. A 4-gene signature associated with clinical outcome in high-grade gliomas. Clin Cancer Res. 2011:17(2):317-27.

28. Vatter $\mathrm{H}$, Seifert V. Ambrisentan, a non-peptide endothelin receptor antagonist. Cardiovasc Drug Rev. 2006;24(1):63-76.

29. Nakashima S, Sugita Y, Miyoshi H, Arakawa F, Muta H, Ishibashi Y, Niino D, Ohshima K, Terasaki M, Nakamura Y, et al. Endothelin B receptor expression in malignant gliomas: the perivascular immune escape mechanism of gliomas. J Neuro-Oncol. 2016;127(1):23-32.

30. Inskip PD, Linet MS, Heineman EF. Etiology of brain tumors in adults. Epidemiol Rev. 1995;17(2):382-414.

31. Jha $P$, Suri $V$, Jain $A$, Sharma $M C$, Pathak $P$, Srivastava A, Suri A, Gupta $D$, Chosdol K, Chattopadhyay $P$, et al. O6-methylguanine DNA methyltransferase gene promoter methylation status in gliomas and its correlation with other molecular alterations: first Indian report with review of challenges for use in customized treatment. Neurosurgery. 2010;67(6):1681-91

32. Rogers SD, Peters CM, Pomonis JD, Hagiwara H, Ghilardi JR, Mantyh PW. Endothelin $\mathrm{B}$ receptors are expressed by astrocytes and regulate astrocyte hypertrophy in the normal and injured CNS. Glia. 2003;41(2):180-90.

33. Cavanagh JB. Corpora-amylacea and the family of polyglucosan diseases. Brain Res Brain Res Rev. 1999;29(2-3):265-95.

34. Libard S, Popova SN, Amini RM, Karja V, Pietilainen T, Hamalainen KM, Sundstrom C, Hesselager G, Bergqvist M, Ekman S et al: Human Cytomegalovirus Tegument Protein pp65 Is Detected in All Intra- and ExtraAxial Brain Tumours Independent of the Tumour Type or Grade. PLoS One. 2014; 9(9):e108861(1-14)

35. Li B, Dewey CN. RSEM: accurate transcript quantification from RNA-Seq data with or without a reference genome. BMC Bioinformatics. 2011;12:323.

\section{Submit your next manuscript to BioMed Central and we will help you at every step:}

- We accept pre-submission inquiries

- Our selector tool helps you to find the most relevant journal

- We provide round the clock customer support

- Convenient online submission

- Thorough peer review

- Inclusion in PubMed and all major indexing services

- Maximum visibility for your research

Submit your manuscript at www.biomedcentral.com/submit

) Biomed Central 